\title{
Archéopages
}

Archéopages

Archéologie et société

Hors-série 1 | 2008

Construction $^{s}$ de l'archéologie

\section{Transgressions et vocations : travail manuel et travail intellectuel en archéologie}

\section{Nathan Schlanger}

\section{(2) OpenEdition}

1 Journals

Édition électronique

URL : https://journals.openedition.org/archeopages/869

DOI : 10.4000/archeopages.869

ISSN : 2269-9872

Éditeur

INRAP - Institut national de recherches archéologiques préventives

Édition imprimée

Date de publication : 1 février 2008

Pagination : 95-97

ISSN : 1622-8545

\section{Référence électronique}

Nathan Schlanger, «Transgressions et vocations : travail manuel et travail intellectuel en

archéologie », Archéopages [En ligne], Hors-série 1 | 2008, mis en ligne le 01 février 2008, consulté le 25 février 2023. URL : http://journals.openedition.org/archeopages/869; DOI : https://doi.org/10.4000/ archeopages. 869 
au point de rejeter la notion d'un groupe à l'origine d'une langue proto-indo-européenne qui aurait donné naissance aux «langues-filles».

Les études les plus récentes (Gray et Atkinson 2003) tendent à obéir au modèle phylogénétique, ou «arborescent». Précisons toutefois que l'on prend de plus en plus conscience des limites de ce modèle «arborescent», par trop simpliste; une approche en «réseau» semble de mieux en mieux admise, car elle propose une alternative aux hypothèses des analyses phylogénétiques (Heggarty 2006). Dans un futur proche, cette tendance pourrait bien prendre la direction souhaitée par Demoule.

Je n'ai qu'un seul reproche à faire à sa position et j'ai eu l'occasion de le lui exprimer de vive voix lors d'une fort agréable rencontre (La Saga des langues indo-européennes) que nous avions animée ensemble à la Cité des Sciences, en 2005. Il se résume au fait que Demoule, à mon avis, n'a pas développé ses idées plus en détail. Sa critique est admirable. Sa vision ne l'est pas moins: «Il faut donc imaginer que les contacts prolongés, pendant des millénaires, de centaines de groupes humains successifs dans l'espace eurasiatique ont évidemment créé, par rencontres, osmoses, emprunts, et parfois aussi conquêtes, les nombreux points de convergence constatés. Il faut donc abandonner ce modèle arborescent, si pauvre et si funeste, pour des hypothèses historiques infiniment plus riches et plus complexes» (Demoule 1999).

Comme esquisse, ce qui précède est remarquable. Mais on aimerait voir cette ébauche prendre forme, ce modèle s'élaborer, parvenir à établir des corrélations plus spécifiques entre les données linguistiques et archéologiques. Elle pourrait reposer sur la théorie des «vagues» de changements linguistiques et jouir du support de l'analyse des réseaux employée avec discernement, afin d'échapper à ces arborescences phylogénétiques qui servent de moule standard à tant d'approches actuelles. Voilà la tâche qui reste à accomplir. Dès que Jean-Paul Demoule se sera libéré de ses fort utiles mais aussi fort prenantes responsabilités actuelles, il pourra retrouver le terrain et peaufiner les détails d'une perspective qu'il a déjà clairement esquissée voilà bientôt trente ans. Sa détermination scientifique, politique et philosophique est, je le crois, intacte.

En reconnaissant tout l'élan et l'intérêt de ce qu'il a déjà apporté dans ce domaine, je dois avouer que l'éloquence de son plaidoyer ne m'a pas encore totalement convaincu. Il m'en faut davantage. J'attends donc avec impatience ses futures œuvres consacrées à cette fascinante et controversée question indo-européenne.

Demoule J.-P. 1980: «Les Indo-Européens ont-ils existé? », L'Histoire, $\mathrm{n}^{\circ}$ 28, p. 109-120.

Demoule J.-P. 1999: «Destin et usage des Indos-Européens », Mauvais temps, no 5, juillet (Éditions Syllepse), p.1-7.

Dixon R. M. W. 1997: The Rise and Fall of Languages, Cambridge, Cambridge University Press.

Gray R. D. et ATKINSON Q. D. 2003: «Language-tree divergence times support the Anatolian theory of Indo-European origin », Nature, $\mathrm{n}^{\circ} 426$, p. 435-439.
Heggarty P. 2006: «Interdisciplinary indiscipline? Can phylogenetic methods meaningfully be applied to language data - and to dating language? ", in P. Forster et C. Renfrew (dir.), Phylogenetic methods and the prehistory of languages, Cambridge, McDonald Institute, p. 183-194.

RENFREw C. 1987 : Archaeology and Language, the Puzzle of IndoEuropean Origins, Londres, Jonathan Cape.

Troubetzkoї N. S. 1939: «Gedanken über das Indogermanenproblem », Acta Linguistica, $\mathrm{n}^{\circ}$ I, p. 81-89, reprinted in A. Scherer (dir.), 1968, Die Urheimat der Indogermanen, Darmstadt, Wissenschaftliche Buchgesellschaft, p. 214-223.

\section{Transgressions et vocations : travail manuel et travail intellectuel en archéologie}

\author{
Nathan Schlanger \\ Inrap
}

$\mathrm{S}$ ous les platanes de la place du village, deux hommes s'attardent [Fig.1]. Celui en blanc, bien à l'aise sur sa chaise pliante, canne au genou et cigarette aux lèvres (ce n'est que vers la quarantaine qu'il a appris à fumer, avec des muletiers du Levante espagnol), écoute ce que lui glisse à l'oreille l'homme en noir, à ses côtés. Un photographe aussi est là, pour prendre le cliché et en confier le tirage (sur carte postale) à l'homme assis, qui l'annote et l'incorpore dans un de ses albums autobiographiques, aujourd'hui déposé aux archives du musée des Antiquités nationales à Saint-Germain-en-Laye.

C'est en retournant cette photo pour en déchiffrer la légende que ce moment de complicité partagée prend les allures d'une anomalie, révélatrice de ce qu'est véritablement l'archéologie. Quelles confidences échangent donc nos deux compères?

Le vieil ouvrier, qui a travaillé avec moi à Gargas en 1912, me raconte qu'il a lu dans Dumont d'Urville que des indigènes de certaines îles se coupent les phalanges en signe de deuil, $\mathcal{E}$ que cela doit expliquer les mains de Gargas à doigts coupés. Gargas 19. IX. 36

Compères? Même si l'abbé Henri Breuil car c'est bien lui, assis, membre de l'Institut et du Collège de France, pape de la Préhistoire au sommet de sa gloire - n'en fait pas état, il y a bien là une infraction manifeste: voilà un ouvrier, anonyme de surcroît, consultant des sources premières pour en énoncer des hypothèses ethnoarchéologiques! Car, chez nos indigènes à nous, du monde occidental, c'est plutôt une division rigide, économique, sociale, voire morale, qui prévaut entre le travail manuel et le travail intellectuel, entre les moyens de production et les œuvres de la raison, entre la matière et l'esprit - un dualisme qui serait même constitutif de notre condition moderne.

Sauf peut-être, justement, en archéologie. En y regardant de plus près, la démarcation entre les champs d'action et de compétence de l'ouvrier et du savant semble n'avoir jamais été si tranchée ou infranchissable. Au cours des siècles, 


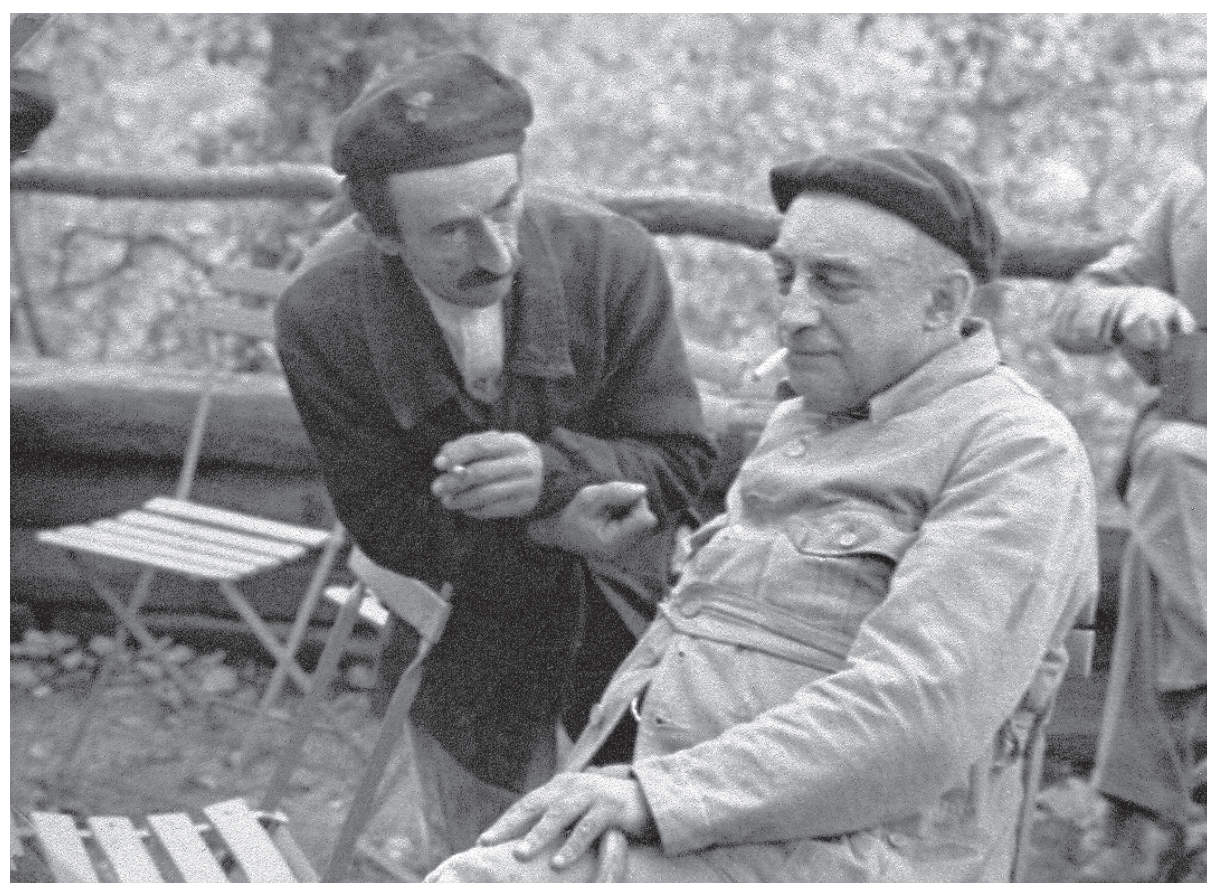

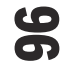

$$
\text { Gaiges ' J. } 1 x \cdot 36
$$

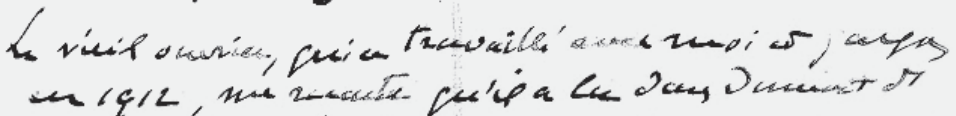

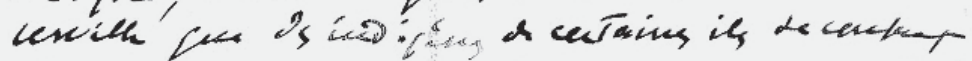

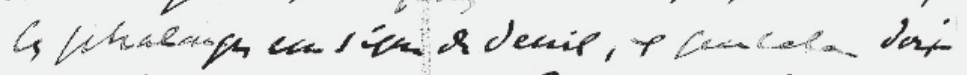

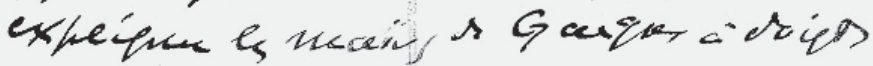
cary'.

$$
\text { ETLSOC ALUHO }
$$


la main-d'œuvre des chantiers archéologiques n'a été que rarement cantonnée au rôle d'exécutant ou de musculature harnachée au service de la science. Sans doute, cette main-d'œuvre a soulevé divers enjeux de rentabilité et de productivité, sans parler de gestion humaine et de contrôle. Boucher de Perthes, précurseur qu'il était, n’a pu échapper à la supercherie de certains de ses ouvriers, qui exhumaient opportunément les faux bifaces qu'ils avaient taillés la veille. D'autres ont mieux su se prémunir: c'est littéralement la jumelle collée à l'œil que l'égyptologue Flinders Petrie arpentait son tell pharaonique pour mieux surveiller les agissements de ses fellahin. De même, il n'a pas échappé à Mortimer Wheeler que sa méthode de fouille par «boxes» de 5 mètres sur 5 permettait de mieux contrôler les fouilleurs, qu'ils appartinssent aux classes laborieuses anglaises ou qu'ils fussent des sujets colonisés.

Mais, en même temps, maints ouvriers se sont investis dans leur gagne-pain en tant qu'êtres humains, avec motivation et fascination. Déjà Boucher de Perthes, grand seigneur, reconnaissait que la sagacité et la persévérance de certains de ses terrassiers leur donnaient lieu de croire qu'ils étaient des savants: "Leur satisfaction était au comble quand, sur, l'étiquette [des pièces qu'ils avaient trouvées] ils apercevaient leur nom. Il en est qui ont refusé le prix d'un morceau en me disant: $<$ Je voudrais seulement qu'on parlât de moi dans le livre `» (Meunier 1875, p. 37). Au-delà donc de l'aspect financier, des ouvriers curieux et dignes de confiance se distinguèrent rapidement auprès des préhistoriens anglais Evans et Prestwich, puis chez le général Pitt Rivers, qui forma sur ses sites plusieurs contremaitres spécialisés. En Orient, c'est en se pliant aux mours locales que Petrie érigea les villageois de Quft en véritable guilde de fouilleurs professionnels. En Afrique subsaharienne, le préhistorien et ethnographe des Mau-Mau, Louis Leakey, sut distinguer, parmi les «boys» qu'il recrutait localement, les plus talentueux, notamment un certain Juma, son meilleur trouveur de fossiles.

Même s'ils ne sortent que peu fréquemment de l'anonymat et reçoivent plus rarement encore le crédit qui leur est dû (et encore, on le voit ici même, plutôt dans les documents et les photos d'archives que dans les publications), ces ouvrierssavants révèlent par leur apparente transgression quelques-unes des spécificités profondes de l'archéologie. À la différence de bien d'autres activités, économiques, techniques mais aussi scientifiques, on peut voir en archéologie une certaine cohérence entre la production du savoir et son contenu. Déjà l'engagement physique du présent, le dispositif d'exhumation, est en quelque sorte en résonance avec la matérialité du passé et de ses vestiges enfouis. De plus, cet engagement se concrétise aisément dans le temps et dans l'espace: sans nier bien évidemment l'importance du laboratoire ni de la bibliothèque, c'est durant les quelques semaines de la campagne de fouille, sur le terrain, que se concentrent les énergies et les consciences. Dans la foulée, savants et ouvriers se côtoient et se salissent en dirigeant leurs efforts communs, intellectuels et manuels, sur les hommes du passé. Et, alors même que se maintient le rythme de travail, que les pelletées s'accumulent et que les brouettes se déchargent sans encombre, il y a dans cet engagement laborieux avec les vestiges du passé humain - des habitations, des inhumations, des restes d'activités quotidiennes, des objets de parure et de prestige, des symboles et des signes (comme, par exemple, des mains à doigts coupés imprimées sur des parois de grottes ornées) - une incitation à la réflexion et au rêve.

Cette vocation est encore plus pertinente dans l'archéologie moderne d'aujourd'hui. Cette archéologie qui s'inspire des principes scientifiques et patrimoniaux de la convention de Malte et qui cherche à contrer l'érosion du passé par une politique de développement durable. Cette archéologie qui sait s'adapter aux échelles et aux échéances de l'aménagement du territoire et remplacer, quand l'occasion le demande, le pénible maniement d'une pelle par le godet lisse d'un engin mécanique. Autant du point de vue interne - celui du métier, de la formation, de la responsabilisation que de celui externe - au niveau des institutions, des opérations, des partenariats -, cette fusion du travail manuel et intellectuel ne fait que confirmer tout l'intérêt que représente l'archéologie, entre science et passion... un antidote à l'aliénation et au désenchantement.

Meunier V. 1875 : Les Ancêtres d'Adam. Histoire de l'homme fossile, Paris, Rothschild éditeur.

\section{Durabilité et archéologie environnementale}

Sander van der Leeuw

Arizona State University

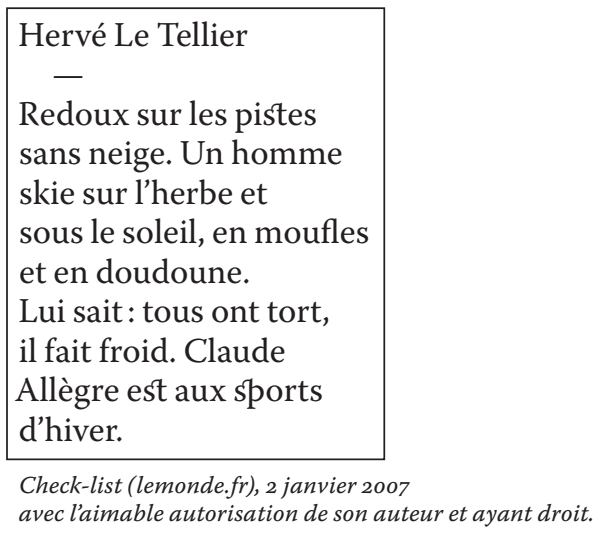

L'environnement, au risque de déplaire... Que cela plaise ou non, force est de constater que notre environnement est en train de changer. L'ouverture, dans le Grand Nord canadien, d'une route maritime reliant l'Europe à l'ExtrêmeOrient n'en est qu'une manifestation parmi 